\title{
Successful management of refractory gastric variceal bleeding via a combined percutaneous approach in sinistral portal hypertension
}

\begin{abstract}
Amongst less common upper gastrointestinal bleeding causes is gastric variceal bleeding due to sinistral portal hypertension. A patient with a history of acute myeloid leukemia and subsequent splenic vein thrombosis presented with melena due to gastric varices bleeding. After failure of medical and endoscopic approach and given that he was not a TIPS candidate, a percutaneous approach was attempted. A transhepatic catheterization and ectopic varices embolization initially stabilized him, but bleeding relapsed. A splenic artery embolization was performed that led to permanent management of the bleeding.
\end{abstract}

Keywords: sinistral portal hypertension, percutaneous transhepatic variceal embolization, splenic artery embolization

Volume 9 Issue 2 - 2018

\author{
Aikaterini Mantaka,' Dimitrios Tsetis, ${ }^{2}$ Adam \\ Hatzidakis, ${ }^{2}$ Elias A Kouroumalis,' Dimitrios \\ N Samonakis' \\ 'Department of Gastroenterology \& Hepatology, University \\ Hospital of Heraklion Crete, Greece \\ ${ }^{2}$ Department of Interventional Radiology, University Hospital of \\ Heraklion Crete, Greece
}

Correspondence: Dimitrios N Samonakis, Department of Gastroenterology \& Hepatology, Building A-4th Floor, University Hospital of Heraklion, Greece, Heraklion 7। I I 0, Tel +302810 392356, Fax +30 2810 542085

Email dsamonakis@gmail.com

Received: December 23, 2017 | Published: March 16, 2018

Abbreviations: SPH, sinistral portal hypertension; SVT, splenic vein thrombosis; TIPS, transjugular intrahepatic portosystemic shunt; B-RTO, balloon-occluded retrograde transvenous obliteration technique; PTVE, percutaneous transhepatic variceal embolization; MRA, magnetic resonance angiography; EUS, endoscopic ultrasound

\section{Introduction}

Gastric varices are present in $20 \%$ of patients with cirrhosis, either in isolation or in combination with esophageal varices. ${ }^{1}$ Although they bleed less frequently than oesophageal varices, the outcome is worse, with high morbidity and mortality. ${ }^{2}$

Sinistral portal hypertension (SPH) is a rare clinical syndrome that leads to gastric varices formation due to splenic vein thrombosis (SVT) in absence of liver function abnormalities or liver cirrhosis. The most common aetiology of SPH is pancreatic disease including cancer, pancreatitis, abscess formation, or a pseudocyst, ${ }^{3-6}$ followed by metastatic carcinoma, retroperitoneal fibrosis, Thrombophilia, ${ }^{7-10}$ pancreatic or splenic lymphoma ${ }^{11}$ and splenic artery malformations ${ }^{12}$ or iatrogenic injury. Gastrointestinal bleeding due to ruptured gastric varices is the most common manifestation of this syndrome which can be life-threatening. ${ }^{13-16}$

Various different treatment approaches have been proposed for gastric variceal bleeding secondary to splenic vein thrombosis. The endoscopic management is the same as in portal hypertension due to cirrhosis. Cyanoacrylate injection achieves haemostasis in more than $90 \%$ of the cases (more than one session may be needed) accompanied by secondary prophylaxis with beta blockers. ${ }^{17}$ However, this approach can either be unsuccessful or not feasible due to inexperienced endoscopist.

Splenectomy is selected for the management of life-threatening, unresponsive to conservative measures variceal bleeding and is performed more often as first choice in SPH in complicated cases of pancreatic disease. ${ }^{4,15}$ Endovascular procedures such as splenic artery embolization $^{18,19}$ and trans-hepatic splenic vein stent placement ${ }^{19}$ have been proved effective, less invasive therapeutic choices in patients unfit for surgical interventions. Another minimally invasive nonsurgical method that directly decompresses portal pressure is transjugular intrahepatic portosystemic shunt (TIPS), although reserved for generalized portal hypertension in cirrhosis. Nevertheless, additional therapy as variceal embolization at the time of the TIPS, may be necessary. ${ }^{20}$ However, not every patient is candidate for TIPS, as multiple medical conditions could be contraindications to it. Embolization of gastric varices has also been achieved using the balloon-occluded retrograde transvenous obliteration technique (B-RTO) via gastrorenal or gastrocaval shunts. Periodic surveillance of these patients is vital since a small increase in portal pressure may cause rebleeding. ${ }^{21}$

Myeloproliferative neoplasms are frequently related to noncirrhotic thrombosis of the splenoportal axis. ${ }^{22}$ Moreover, patients with myelofibrosis and myeloid metaplasia can present with portal hypertension. ${ }^{23}$ We report a patient with a history of myelofibrosis and reccurent thrombotic episodes, presenting with massive gastric variceal bleeding caused by SPH. We describe the diagnostic and therapeutic approach to this patient and review of the literature.

\section{Case presentation}

A 70-year-old man was admitted to the emergency department due to repeated episodes of melena over the last 10 days and progressive fatigue. The patient had a history of thrombophilia (deep vein thrombosis, pulmonary embolism with filter in inferior vena cava), myelofibrosis progressed into acute myeloid leukemia, severe aortic 
stenosis and heart failure, diabetes mellitus and mild renal failure. He had no bleeding episodes in the past. He was already anticoagulated with asenocoumarol (INR:1.61) and on antiplatelet therapy with acetylsalicylic acid.

On admission the patient was pale, with low blood pressure BP:85/50mmHg, tachycardia HR:95/min and his laboratory tests revealed severe anemia $\mathrm{Ht}: 17.8 \%, \mathrm{Hb}: 5.2 \mathrm{~g} / \mathrm{dl}, \mathrm{MCV}: 77.7 \mathrm{fl}$, RDW:24\%, renal failure (Urea:188mg/dl, Creatinine:1.6mg/dl), hyperkalemia (K:5.6mEq/1, normal range:3.5-5) with normal liver function tests and INR:1.4. After hemodynamic stabililization and transfusion of 6 units of red blood cells and 2 units of fresh frozen plasma, an emergency endoscopy was performed. Large bleeding fundal varices (GOV-2 \& isolated) were revealed. Histoacryl was not available at that time and injection sclerotherapy with ethanolamine was attempted. Large thrombus was formed but varices went on oozing. He was commenced on i.v. antibiotics and somatostatin. Splenic vein occlusion/thrombosis was accordingly suspected. An abdominal ultrasound showed enlarged spleen $17 \mathrm{~cm}$, with normal liver parenchyma (without any signs of liver cirrhosis), patent portal vein but there was no flow in the splenic vein.

Due to failure of the medical and endoscopic treatment to control

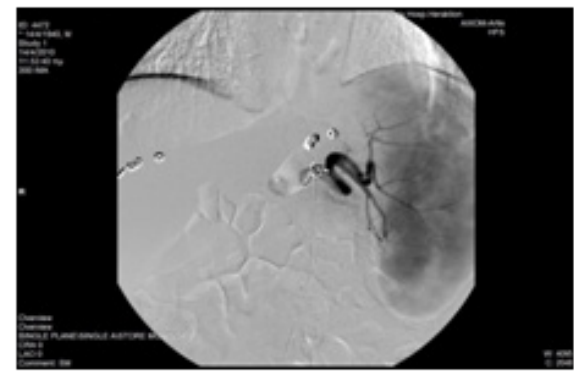

Figure IA Selective arteriography of the spleen showing normal arterial vascular tree on a enlarged spleen. The coils from the previous emergency percutaneous transhepatic variceal embolization of the patient's ectopic veins are observed.

He was discharged 2 weeks post splenic artery embolization, clinically improved. Repeat MRI scan of the abdomen 2 months later, revealed liquefaction of splenic parenchyma with high solid content and peripheral enhancement after intravenous injection of contrast medium. These imaging findings were indicative of possible inflammatory content in the liquefied part of the spleen. Six months later spleen was still liquefied, with a substantial reduction in size from $17 \mathrm{~cm}$ down to $11 \mathrm{~cm}$ on MRI. No further bleeding episodes were noted after one year of follow up although the patient was repeatedly hospitalized due to complications of cardiac and haematological comorbidities.

\section{Discussion}

Non-cirrhotic gastric variceal bleeding results from significant increase in pressure of the left-sided portal circulation. It is important, but not always easy, to differentiate between sinistral and classical portal hypertension since the optimal management is quite different. Thrombotic occlusion of the splenic vein is the primary cause of SPH with pancreatic diseases being the main source of such thrombosis. Myeloproliferative neoplasms account for the majority of noncirrhotic and non-malignant splanchnic vein thrombosis. bleeding, the patient was again tachycardic with hypotension (HR:98/ min, BP:90/55mmHg), a percutaneous transhepatic approach was decided as he was unsuitable for TIPS due to cardiac co-morbidities. During selective venography, the splenic vein was found totally occluded and multiple ectopic fundal varices (collaterals) were identified. An emergency percutaneous transhepatic variceal embolization (PTVE) of the patient's ectopic veins with coils was performed without serious side effects. One week after the ectopic varices embolization the patient had a repeated episode of hematemesis with hemodynamic destabilization (BP:80/45mmHg, HR:105/min). A Sengstaken-Blakemore tube was inserted and a repeat endoscopy revealed multiple fundic varices with cherry red spots, an ulcer on a varix surface and blood clots in the stomach. Blood transfusion with 4 units of red blood cells was given.

At this point we proceeded to an emergency selective embolization of splenic artery with embospheres (more than $70 \%$ of the total splenic volume was embolized) (Figure 1A) (Figure 1B). The procedure was well tolerated and proved successful; there was no recurrence of bleeding post-embolization. Mild analgesics were given for left upper abdominal pain and antibiotic administration was continued. Due to peripheral oedema, ascites, pleuritic and pericardial effusion the patient was commenced on diuretic therapy and nutritional support.

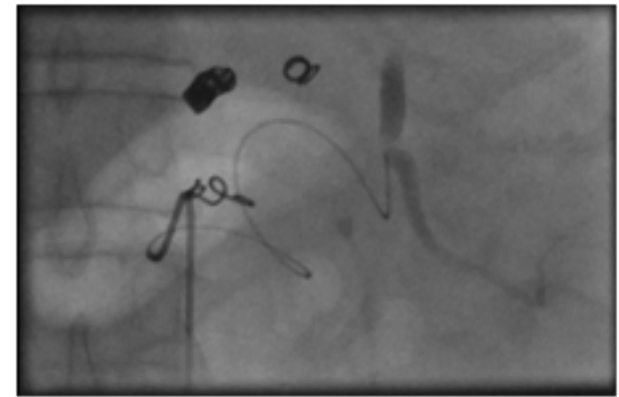

Figure IB Following embolization with embosphere particles through a microcatheter, angiographic abolishment of peripheral splenic arterial branches is observed.

Diagnostic imaging plays an important role in confirming splenic vein occlusion, but in cases of severe upper gastrointestinal bleeding, hemostasis is the primary goal. Trans-abdominal ultrasonography and doppler ultrasonography of the splenoportal axis is usually used to differentiate between systemic versus sinistral portal hypertension. When transabdominal ultrasound is non-diagnostic, other modalities as endoscopic ultrasound (EUS), CT scan, magnetic resonance angiography (MRA) could have better accuracy. ${ }^{24,25}$ Undoubtfully, the gold standard in diagnosing sinistral portal hypertension is angiography.

Fundic variceal bleeding can be life-threatening ${ }^{26}$ and direct intervention is more than crucial. Endoscopic approaches or TIPS used as treatment for refractory haemorrhage from varices in cirrhotic patients, show marginal benefit in sinistral hypertension. Therapeutic choices for bleeding control include injection therapy (tissue adhesives) and less often band ligation as first-line endoscopic therapies..$^{27,28}$ More sophisticated interventional radiologic techniques and splenectomy are selected especially in cases unresponsive to conservative management. However, the long-term rebleeding rate after endoscopic cyanoacrylate injection is still high ${ }^{1}$ in addition to potential risk of systemic migration through the inferior vena cava. 
Bhat et $\mathrm{al}^{29}$ have reported successful, EUS-guided, treatment of gastric fundic varices with combined injection of coils and cyanoacrylate in 152 patients, with a low re-bleeding rate of $3 \%$ during long-term follow-up.

PTVE using coils and/or sclerosing agents has been an alternative therapeutic option, significantly lowering variceal rebleeding rate due to a wider obliteration range of collaterals in the vicinity of the gastric fundus. ${ }^{30}$ This technique has been widely used either alone ${ }^{30}$ or in combination with TIPS ${ }^{31}$ or B-RTO ${ }^{32}$ in cases of refractory variceal bleeding in cirrhotic patients. PTVE does not always lead to complete eradication of gastric varices, probably due to the presence of multiple inflow vessels and has limitations in cases of ascites. Furthermore, the underlying portal hypertension remains unaffected and recurrent bleeding has been reported in several cases within a few months after embolization. ${ }^{33}$ For this reason, PTVE is considered as an adjuvant rather than radical treatment. ${ }^{34,35} \mathrm{~B}-\mathrm{RTO}$ is an invasive endovascular technique for gastric varices obliteration, using a sclerosing agent injected through a balloon catheter. Simultaneous obliteration of the gastrorenal shunt is necessary for preventing bleeding recurrence. ${ }^{36}$

In refractory cases, splenic artery embolization by selective catheterization is an option for patients who are not medically fit for a splenectomy. Wang et al..$^{37}$ described 14 cases with sinistral portal hypertension due to pancreatic diseases, and treated with splenic artery embolization, mostly for bleeding complications. The approach was feasible and effective both clinically and radiologically. Similar approach is proposed in a previous report for patients with poor performance status and sinistral portal hypertensive bleeding. ${ }^{38}$

Data are lacking on the role of endovascular portal vein stenting for the management of sinistral portal hypertension. ${ }^{7}$ On the contrary, splenectomy has been proved to be an effective alternative treatment option for SPH due to splenic vein thrombosis, minimizing variceal rebleeding rates, although surgery has many limitations in patients with multiple co- morbidities.

In our case a combination of selective transhepatic variceal embolization with coils followed by splenic artery embolization with embospheres, was performed in order to control recurrent gastric variceal bleeding in a high-risk patient with splenic artery thrombosis and multiple co-morbidities. The approach was effective in the long term with minimal side effects and can be considered in similar cases.

\section{Acknowledgements}

Aikaterini Mantaka: wrote the paper and contributed to patient management, Dimitrios Tsetis: performed spleenic artery embolization, Adam Hatzidakis: performed percutaneous embolization, Elias Kouroumalis: supervised patient treatment and paper writing, Dimitrios Samonakis: contributed to patient management and follow up and to paper writing

\section{Conflict of interest}

Authors have no conflict of interest to declare.

\section{Patient consent}

Patient signed an informed consent and gave his permission to publish the related medical data.

\section{References}

1. Garcia-Tsao G, Bosch J. Management of Varices and Variceal Hemorrhage in Cirrhosis. N Engl J Med. 2010;362(9):823-832.

2. Sarin SK, Lahoti D, Saxena SP, et al. Prevalence, classification and natural history of gastric varices: a longterm follow-up study in 568 portal hypertension patients. Hepatology. 1992;16(6):1343-1349.

3. Singhal D, Kakodkar R, Soin AS, et al. Sinistral portal hypertension: a case report. JOP. 2006;7(6):670-673.

4. Little AG, Moossa AR. Gastrointestinal hemorrhage from left-sided portal hypertension. An unappreciated complication of pancreatitis. Am J Surg. 1981;141(1):153-158.

5. Manenti A. Splenic vein obstruction secondary to pancreatic carcinoma Acta Chir Belg. 1981;80(5):245-248.

6. Keith RG, Mustard RA, Saibil EA. Gastric variceal bleeding due to occlusion of splenic vein in pancreatic disease. Can J Surg. 1982; 25(3):301-304.

7. Köklü S, Yüksel O, Arhan M, et al. Report of 24 left-sided portal hypertension cases: a single-center prospective cohort study. Dig Dis Sci. 2005;50(5):976-982.

8. Honda Y, Ueda M, Kyoi M, et al. An unusual case of portasystemic encephalopathy caused by splenic vein occlusion following gastrectomy. Am J Gastroenterol. 1978;69(5):590-593.

9. Koehler RE. Case: splenic vein obstruction due to metastatic hypernephroma. Gastrointest Radiol. 1981;6(4):365-370.

10. Lavender S, Lloyd-Davies RW, Thomas ML. Retroperitoneal fibrosis causing localized portal hypertension. Br Med J. 1970; 3(5723):627628.

11. Chen BC, Wang $\mathrm{HH}$, Lin $\mathrm{YC}$, et al. Isolated gastric variceal bleeding caused by splenic lymphoma-associated splenic vein occlusion. World J Gastroenterol. 2013:28;19(40):6939-6942.

12. Beksac K, Karakoc D. Multiple giant splenic artery aneurysms causing sinistral (left-sided) portal hypertension. M Case Rep Gastrointest Med. 2016;2016:6278452

13. Bradley EL. III: The natural history of splenic vein thrombosis due to chronic pancreatitis: indications for surgery. Int $J$ Pancreatol. 1987;2:87-92.

14. Lillemoe KD, Yeo CJ. Management of complications of pancreatitis. Curr Probl Surg. 1998;35:1-98.

15. Evans GR, Yellin AE, Weaver FA, et al. Sinistral (left-sided) portal hypertension. Am Surg. 1990;56(12):758-763.

16. Alwmark A, Gullstrand P, Ihse I, et al. Regional portal hypertension in chronic pancreatitis. Acta Chir Scand. 1981; 147(2):155-157.

17. Sarin SK, Kumar A. Endoscopic treatment of gastric varices. Clin Liver Dis. 2014;18(4):809-827.

18. Thompson R, Taylor M, McKie L, et al. Sinistral Portal Hypertension. Ulster Med J. 2006;75(3):175-177.

19. Cakmak O, Parildar M, Oran I, et al. Sinistral Portal Hypertension; imaging findings and endovascular therapy. Abdom Imaging. 2005;30(2):208-213.

20. Boyer TD, Haskal ZJ. AASLD Practice Guideline. The role of transjugular intra-hepatic portosystemic shunt in the management of portal hypertension. Hepatology. 2005;41(2):386-400. 
21. Saad WE. Balloon-occluded retrograde transvenous obliteration of gastric varices: concept, basic techniques, and outcomes. Semin Intervent Radiol. 2012;29(2):118-128.

22. Hoekstra J, Bresser EL, Smalberg JH, et al. Long-term follow-up of patients with portal vein thrombosis and myeloproliferative neoplasms. J Thromb Haemost. 2011;9(11):2208-2214.

23. Abu-Hilal M, Tawaker J. Portal hypertension secondary to myelofibrosis with myeloid metaplasia: a study of 13 cases. World J Gastroenterol. 2009;15(25):3128-3133.

24. Lewis JD, Faigel DO, Morris JB, et al. Splenic vein thrombosis secondary to focal pancreatitis diagnosed by endoscopic sonography. $J$ Clin Gastroenterol. 1998;26(1):54-56.

25. Kreft B, Strunk H, Flacke S, et al. Detection of thrombosis in the portal venous system: comparison of contrast-enhanced MR angiography with intraarterial digital substraction angiography. Radiology. 2000;216(1):86-92.

26. Dagradi AE, Siemsen J, Brook J, et al. Bleeding esophageal varices in myelofibrosis. Am J Gastroenterol. 1965;44(6):536-544.

27. de Franchis R, Baveno V. Faculty Revising consensus in portal hypertension: report of the Baveno $\mathrm{V}$ consensus workshop on methodology of diagnosis and therapy in portal hypertension. $J$ Hepatol. 2010;53(4):762-768.

28. Sarin SK, Jain AK, Jain M, et al. A randomized controlled trial of cyanoacrylate versus alcohol injection in patients with isolated fundic varices. Am J Gastroenterol. 2002;97(4):1010-1015.

29. Bhat YM, Weilert F, Fredrick RT. EUS-guided treatment of gastric fundal varices with combined injection of coils and cyanoacrylate glue: a large U.S experience over 6 years (with video). Gastrointest Endosc. 2016;83(6):1164-1172.

30. Wang J, Tian XG, Li Y, et al. Comparison of modified percutaneous transhepatic variceal embolization and endoscopic cyanoacrylate injection for gastric variceal rebleeding. World $J$ Gastroenterol. 2013;19(5):706-714

31. Tesdal IK, Filser T, Weiss C, et al. Transjugular intrahepatic portosystemic shunts: adjunctive embolotherapy of gastroesophageal collateral vessels in the prevention of variceal rebleeding. Radiology. $2005 ; 236(1): 360-367$

32. Uchiyama F, Murata S, Onozawa S, et al. Management of gastric varices unsuccessfully treated by balloon-occluded retrograde transvenous obliteration: long-term follow-up and outcomes. Scientific World Journal. 2013;2013:498535.

33. L'Herminé $\mathrm{C}$, Chastanet $\mathrm{P}$, Delemazure $\mathrm{O}$, et al. Percutaneous transhepatic embolization of gastroesophageal varices: results in 400 patients. AJR Am J Roentgenol. 1989;152(4):755-760.

34. Scott J, Dick R, Long RG, et al. Percutaneous transhepatic obliteration of gastro-oesophageal varices. Lancet. 1976;2(7976):53-5.

35. Keller FS, Dotter CT, Rosch J. Percutaneous transhepatic obliteration of gastroesophageal varices: some technical aspects. Radiology. 1978;129(2):327-32.

36. Katoh K, Sone M, Hirose A, et al. Balloon-occluded retrograde transvenous obliteration for gastric varices: the relationship between the clinical outcome and gastrorenal shunt occlusion. BMC Med Imaging. 2010;10:2.

37. Wang Q, Xiong B, Zheng C, et al. Splenic Arterial Embolization in the Treatment of Severe Portal Hypertension Due to Pancreatic Diseases: The Primary Experience in 14 Patients. Cardiovasc Intervent Radiol. 2016;39(3):353-358.

38. Liu Q, Song Y, Xu X, Jin Z, Duan W, Zhou N. Management of bleeding gastric varices in patients with sinistral portal hypertension. Dig Dis Sci. 2014;59(7):1625-1629. 